\title{
Effective Compression of Quantum Braided Circuits Aided by ZX-Calculus
}

\author{
Michael Hanks@ ${ }^{1, *}$ Marta P. Estarellas $\odot,{ }^{1, *}, \dagger$ William J. Munro®, ${ }^{2,1}$ and Kae Nemoto® ${ }^{1}$ \\ ${ }^{1}$ National Institute of Informatics, 2-1-2 Hitotsubashi, Chiyoda-ku, Tokyo 101-8430, Japan \\ ${ }^{2}$ NTT Basic Research Laboratories \& NTT Research Center for Theoretical Quantum Physics, \\ NTT Corporation, 3-1 Morinosato-Wakamiya, Atsugi, Kanagawa 243-0198, Japan
}

(Received 31 January 2020; revised 23 May 2020; accepted 1 September 2020; published 11 November 2020)

\begin{abstract}
Mapping a quantum algorithm to any practical large-scale quantum computer will require a sequence of compilations and optimizations. At the level of fault-tolerant encoding, one likely requirement of this process is the translation into a topological circuit, for which braided circuits represent one candidate model. Given the large overhead associated with encoded circuits, it is paramount to reduce their size in terms of computation time and qubit number through circuit compression. While these optimizations have typically been performed in the language of three-dimensional diagrams, such a representation does not allow an efficient, general, and scalable approach to reduction or verification. We propose the use of the $\mathrm{ZX}$-calculus as an intermediate language for braided circuit compression, demonstrating advantage by comparing results using this approach with those previously obtained for the compression of $|A\rangle$ and $|Y\rangle$ state distillation circuits. We then provide a benchmark of our method against a small set of Clifford $+T$ circuits, yielding compression percentages of $\sim 77 \%$. Our results suggest that the overheads of braided, defect-based circuits are comparable to those of their lattice-surgery counterparts, restoring the potential of this model for surface-code quantum computation.
\end{abstract}

DOI: $10.1103 /$ PhysRevX.10.041030

Subject Areas: Quantum Information

\section{INTRODUCTION}

Quantum computers hold the promise of finding solutions to problems that cannot be efficiently treated using the general classical model of computation [1-3]. Given the potential advantages this technology has to offer, and its latest developments [4], global efforts are currently focusing on the design of practical large-scale quantum computer architectures that will allow for a real quantum advantage [5]. On the software side, a common element to those designs is the need to specify a quantum compilation process. As in classical computers, a complete stack of consecutive translations is needed in order to map an arbitrary quantum algorithm into a reduced set of machine operations (see Fig. 1). We identify that the general question of parsing a quantum problem from a high-level description into machine language for large-scale applications needs to involve at least three compilations. At the highest level of abstraction, the quantum algorithm is written in a human-readable language and the first

\footnotetext{
*These authors contributed equally to this work. †mpestarellas@nii.ac.jp
}

Published by the American Physical Society under the terms of the Creative Commons Attribution 4.0 International license. Further distribution of this work must maintain attribution to the author(s) and the published article's title, journal citation, and DOI. compilation generates a quantum circuit from a reduced set of universal quantum gates. The second compilation is in charge of making the computation robust to errors and thus requires the inclusion of quantum error correction methods. At the lowest level, the fault-tolerant circuit has to be translated into a sequence of hardware-specific classical instructions (e.g., sequence of microwave pulses) that leads the machine to effectively perform the desired computation.

A major challenge in the realization of a large-scale quantum computer is related to the redundancy required by the error-correction codes and their need of large numbers of physical qubits. One of the most promising error-correcting codes, offering accuracy threshold values of $\sim 1 \%$, is the surface code [6-8]. Depending on the way the logical qubits are encoded in the surface code, the type of operations applied to the physical qubits will differ leading to two main models of computation: braiding (defect-based encoding) [9] and lattice surgery (planarbased encoding) [10]. Each of these is represented as a different 3D diagram that keeps track of the operations (braiding, deformation, and merging or splitting) that need to be applied between the logical degrees of freedom of each representation. Given that for large-scale purposes any arbitrary optimized non-error-corrected circuit will likely need to be translated into a topological one, the optimization of such intermediate circuit will be paramount in order to reduce its associated resources and therefore relax the physical demands of the quantum device. 


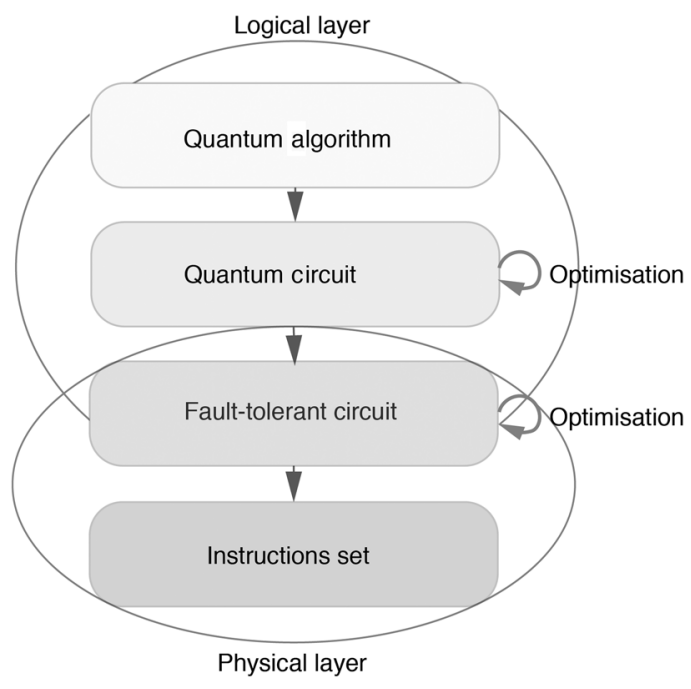

FIG. 1. General compilation stack of a large-scale quantum computer architecture.

Motivated by the search for low-overhead error-corrected circuits to bring forward the regime of quantum practicality, we here focus on the compression of defectbased topological quantum circuits. Given such a circuit, we want to reduce the resources associated with the circuit volume, i.e., the time and number of defects (and, therefore, physical qubits). These optimizations have typically been performed in the language of three-dimensional diagrams [9,11]. However, due to the complicated nature of large circuits expressed in this representation, it is difficult to intuit and keep track of transformation rules. Importantly, this representation provides no strategy to ensure the independence of errors arising from different local gates in distillation circuits or to verify such circuits beyond Clifford-group subsegments. We here instead propose the use of the ZX-calculus representation [12] as an alternative language for braided topological circuit optimization. The ZX-calculus clarifies our intuition about topological transformations and provides a straightforward method for the verification of error independence and therefore fault tolerance [13]. Importantly, we show how the primitive elements of the ZX-calculus can be mapped directly to elements of a topological circuit each requiring specified resources. Therefore, using this route we can optimize the circuit with the ZX-calculus and then convert back to the 3D representation to apply final arrangements and trivial transformations for a further packing of the braids. We demonstrate how this approach allows the compression of general braided circuits, observing reductions of up to $\sim 77 \%$. We also demonstrate that the resources required for these reduced braided structures are comparable to those obtained for circuits based on lattice surgery [14].

Following an introduction to braided circuits (Sec. II) and the ZX-calculus (Sec. III), we demonstrate the use of the ZX-calculus as an intermediate language for circuit reduction with two exemplar magic state distillation circuits in Sec. IV. We next present a wider range of compression results and discuss the potential of hybrid braid and latticesurgery approaches in Secs. V and VI, respectively. Finally, in Sec. VII we discuss the implications of our results in the broader context of quantum circuit compilation.

\section{BRAIDED CIRCUITS}

The surface code [6-8] is one of the most promising error-correction codes for large-scale quantum applications. In this code the physical qubits are arranged in a meshlike 2D lattice and are divided into two types: data qubits and ancillas. Data qubits are identified with edges of the square lattice and the ancillas are identified with either vertices or faces (sometimes named plaquettes). Parity measurements are repeatedly performed at the ancillas to identify and track errors occurring in the data qubits. The structure of the code is similar to the structure of the letter $\mathrm{H}$; on two sides (left and right) we have boundary vertices with only three incident edges, while on the remaining two sides (top and bottom) we have the remnants of face operators from which one boundary edge has been removed. The former we call smooth boundaries and the latter rough. Vertices and faces are stabilized by Pauli operators defined in orthogonal bases, respectively, such that they form two codes that together protect from any arbitrary single-qubit error. Such a lattice is capable of encoding one logical qubit fault tolerantly, but to perform any computation one needs to increase the logical degrees of freedom. Here we focus on the braided defect model as a surface-code-based strategy for fault-tolerant quantum computation.

\section{A. Defect qubits}

In the braided defect model additional logical degrees of freedom are introduced by opening holes in the surface of the lattice such that the number of logical qubits is increased by one. When we introduce multiple holes (or defects) into the lattice, typically we do not make use of the entire code space. Instead, we restrict the available logical operations so that we can identify qubits locally with particular defect pairs. Local defect qubits can be constructed with boundaries of either type (smooth or rough). We call defects with one type of boundary primal and the other dual, and the bases of their respective logical operators are inverted.

Defects in the lattice of a surface code can be expanded by measuring physical qubits across a region contiguous with the defect [5], in the basis of the stabilizer operations around the defect boundary. This will identify string operators crossing the expanded region, and all such strings are corrected outward to surround the new boundary. Similarly, defects can be contracted by initializing qubits and resuming stabilizer measurements in a region 
contiguous with the defect boundary. The correction may then proceed as if all qubits in the new region had been lost [15-17]. With the ability to expand and contract, we are able to migrate defects about the lattice. Such migration allows us to implement nonlocal braiding operations [18]. With braiding, string operators associated with one defect pair can be made to wrap around a pair with a different boundary type (see enlargement at the bottom of Fig. 2). The effect of these two-qubit string operator correlations is to implement a CNOT gate. Care must be taken to maintain the code distance during migration.

The mechanics of defect migration can also be applied to split and merge defects, in a manner analogous to lattice surgery [10]. Any string operator surrounding a defect,
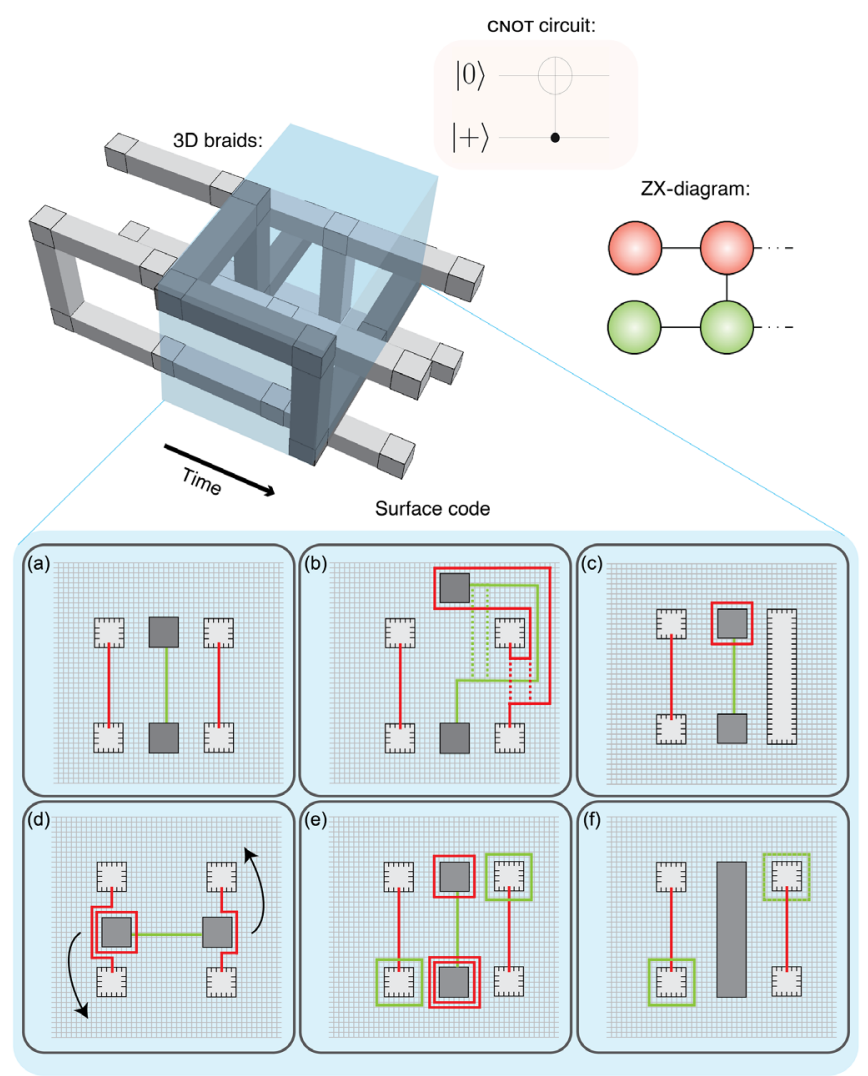

FIG. 2. Example of a small quantum circuit performing a CNOT gate in the diagram, 3D and ZX-calculus representations. Bottom, snapshots of the braiding process (note corrections are not shown). (a) Two primal qubits initialized in the $|+\rangle$ and $|0\rangle$ states, and a dual qubit, initialized in the $|+\rangle$ state (the initial split operation for the dual qubit initialization is omitted). (b) The dual defect braids with the first primal qubit. (c) A merge operation between the two defects of the first primal qubit projects its state onto the dual qubit, and the merged defect is used to reinitialize the primal qubit in the $|0\rangle$ state. (d),(e) Braided interaction between the dual qubit and both primal qubits performs a CNOT gate with two targets. (f) A merge operation between the two defects of the dual qubit measures it in the $\mathrm{X}$ basis, so that the total operation corresponds to a CNOT from the first primal qubit to the second. while a subregion of parity measurements is reinitialized across the diameter, will necessarily surround both resulting smaller defects. Similarly, any string operator in contact with the boundary of a defect while such a subregion is reinitialized will be in contact with one of the two smaller defects. Conversely, when the migration of two defects brings them together, the measurement of individual qubits between the two defects will identify the parity of string operators crossing between them. String operators in contact with one of two merging defects will of course remain in contact with the larger composite region. In fact, the extension of a defect itself can be viewed as a merge between an existing logical qubit and a newly created logical qubit in a superposition state, while a split has all the mechanics of contraction. These merge and split operations are typically used for single-qubit initialization and measurement, but can also be applied between qubits. The direction of corrective operations from the central subregion between two defects will be a matter of convention.

\section{B. 3D representation}

Three-dimensional diagrams are typically used to represent the behavior of defects opened in the surface code as they evolve across time. In such representations, we ignore the individual physical qubits that make up the surface-code lattice, and represent the defect regions as pipes of appropriate relative width $[11,18]$. These pipes are either light (primal) or dark (dual), and wind around one another in three dimensions such that a two-dimensional cross section of the structure displays the locations of the defect regions at a point in time. In Fig. 2 we show a braided CNOT gate between two primal qubits (with a dual qubit as intermediary).

Verification of 3D structures is performed via "correlation surfaces" [19], which track the evolution of logical operators across the structure in time. This method does not distinguish between circuits that differ only by topologypreserving transformations, nor does it track the many possible phase combinations arising from probabilistic teleported gates. The final state may be preserved, conditional on the outcomes of gate teleportation, and it is possible to eliminate certain local operations (including measurements), without noticing the effect on higher encoded logical layers (as in distillation circuits). We encounter precisely this issue when comparing prior results for the $Y$ - and $A$-state distillation circuits in Sec. IV: the former appears to have weakened the protective structure of the distillation circuit, while the latter has been limited at the positions of $T$-gate injections by a desire to conserve it.

No efficient method of complete verification exists for arbitrary quantum circuits. However, in the case of the 3D representation we are faced with the further difficulty that the very definition of qubits can be ambiguous. Looking ahead to Sec. IVA, we observe that there are many ways to define qubits between defects in a 
3D structure. When attempting to reconstruct a circuitlike representation, many choices lead to counterintuitive results-such as the measurement and destruction of "half" a qubit. These results can ultimately be reconciled in terms of the equivalence between entanglement and the destruction of logical degrees of freedom. However, it remains difficult to interpret reduced 3D structures as circuit diagrams and takes some significant effort just to identify what is happening in the language of qubits and subcircuits, let alone to verify the entire computation.

\section{Circuit reduction}

As a consequence of the tremendous progress in quantum technologies over the past decade, efforts have now focused on the reduction of the resources associated with large error-corrected quantum circuits. We may divide topological circuit reduction into three categories: gate efficiency, static reduction, and dynamic reduction. The first, gate efficiency, involves the discovery of new and more efficient methods for implementing quantum gates on encoded information. Examples of this first type are measurements based on merging and splitting [5], as opposed to isolating regions of the lattice as if they were independent planar codes [20]. Other examples are the direct implementation of the phase and Hadamard gates [21], or the implementation of the CNOT gate, which may be performed via braiding, as mentioned above, but has also been performed via teleportation-inspired "junctions" [22]. The second type, static reduction, is circuit reduction in its usual form; given a set of gates and transformation rules or identities, we seek to rearrange the elements of the circuit so that it occupies a smaller resource-time volume. Examples are the direct "compaction" of defect pipes [11], the use of "bridging" to eliminate redundant portions of the circuit [9], or the selective arrangement of the components of magic state distilleries [23]. The dynamic element of circuit reduction, in contrast to the static element, seeks less to reduce any single circuit and more to find an approach that can be applied to any circuit to reduce the overhead costs associated with information received at run-time. One example is the use of online scheduling [24], while another is the use of selective-route teleportation [25] to fix the time cost of a circuit with probabilistic elements.

One approach that has been used to simplify the reduction (and partial verification) of topological quantum circuits is the ICM representation [26]. This representation first divides the circuit into the three distinct phases of "initialization," "CNOT gates," and "measurement." Once this has been achieved, and making use of selective-route teleportation to fix the form of the circuit, the CNOT-restricted segment of the circuit can be reduced and verified with "stabilizer" [27] and "correlationsurface" methods [19].
We here restrict ourselves to the static reduction of general quantum circuits; we will not make use of the ICM representation or related techniques on restricted circuit classes. As in previous works, we assess the circuit compression in terms of reducing the volume of an imaginary bounding box that surrounds the 3D structure. This volume, in units of the code distance $d$, has a direct correspondence with both the number of necessary physical qubits and the total computation time.

\section{ZX-CALCULUS}

The ZX-calculus is a tensor-network-based diagrammatic language introduced by Coecke and Duncan [28,29] that allows for a high-level and intuitive representation of pure state qubit quantum processes. This representation is universal and complete for Clifford $+T$ quantum mechanics $[30,31]$, of direct concern to surface-code quantum computing, and provides a complete set of rewrite rules for reasoning about equivalent diagrams. The application of the ZX-calculus has proven fruitful in the representation of surface codes with lattice surgery [32], for design and analysis of topological quantum algorithms [33], and as a method for reducing the number of $T$ gates in a quantum circuit [34].

By convention, ZX-diagrams are read timewise from left (inputs) to right (outputs). They are connected, undirected graphs, with edges representing single qubits and labeled green (red) nodes representing transformations and measurements of these qubits in the $\mathrm{Z}(\mathrm{X})$ basis (see Fig. 3).

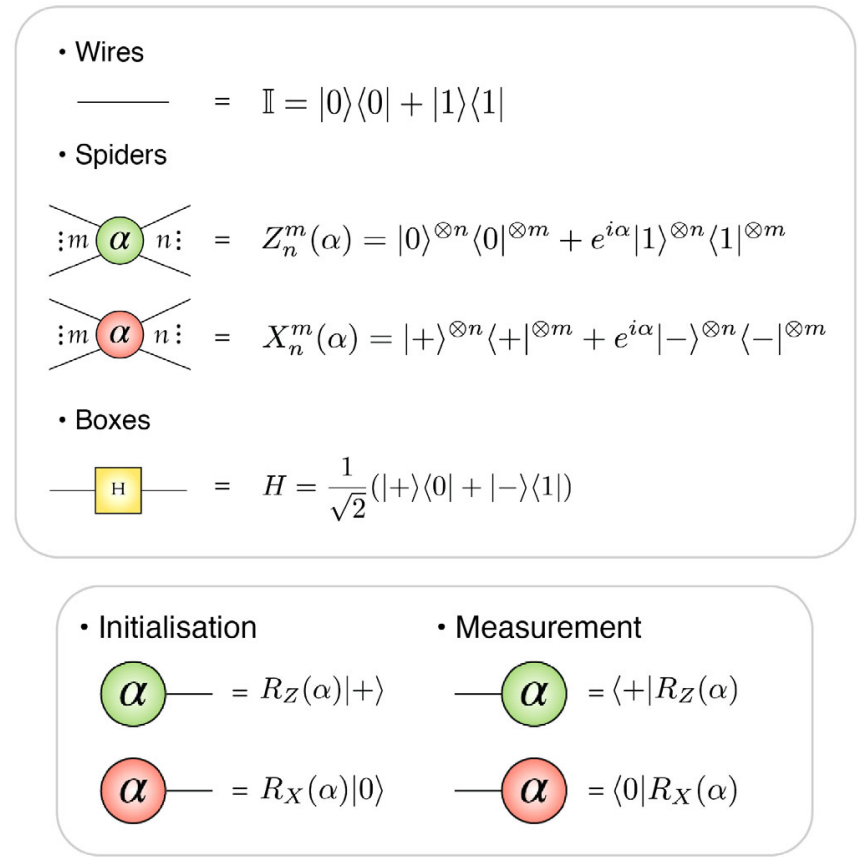

FIG. 3. ZX-calculus elements and their associated linear operations. Initialization and measurement operations in the $\mathrm{Z}$ and $\mathrm{X}$ basis. 


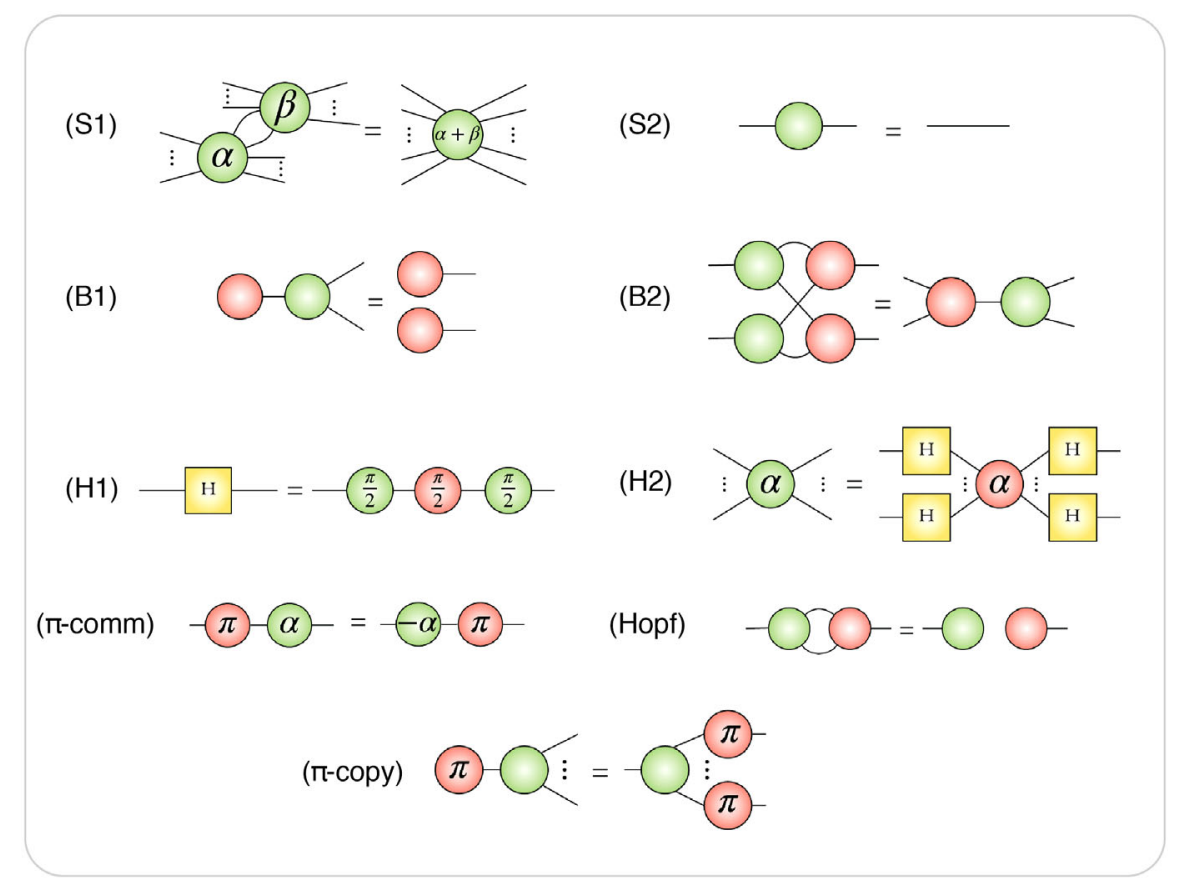

FIG. 4. ZX-calculus rules. This set of reversible axioms or rewrite rules is complete for Clifford circuits.

Nodes may have many edges and are referred to as spiders, and additionally a yellow box is used as shorthand to represent the single-qubit Hadamard gate. Placing elements above and below one another denotes their tensor product $(\otimes)$, while connecting nodes denotes their composition (o). The circuit is preserved under any bending, stretching, or twisting of wires; nodes may be rearranged as long as the elements and connectivity of the diagram are conserved (graph isomorphism). Qubit initialization and projective measurement are common operations represented as shown in Fig. 3.

In Fig. 4 we outline the axioms needed for Clifford completeness. Each of these rewrite rules holds under the exchange of red and green nodes or when the diagrams are reflected. The original axioms include disconnected components ("scalars") to normalize equivalent structures [35]. These scalars do not affect the connectivity of the diagram, and we may therefore ignore them in our resource estimates. However, it should be remembered that probabilistic elements and associated corrections remain present.

Our approach to compression relies on the translation of our circuit into this graph representation. The initial translation is straightforward when the circuit is expressed in Clifford $+T$ form. Following this, the reduction of a $\mathrm{ZX}$-calculus graph consists of the application of a sequence of rewrite rules (see Fig. 4) to minimize the number of red, green, and yellow nodes. When dealing with braided circuits, this minimization has two additional constraints: (1) we want to minimize the number of $\pi / 4$ and $\pi / 8$ gates ( $S$ and $T$ gates, respectively), as their presence has overhead associated with distilleries (described in Sec. IV), and
(2) we want to minimize the number of Hadamard gates, also due to their additional overheads. The ZX-graph reduction of small circuits, such as those we present in Sec. IV, can be easily done by hand. In contrast, for larger circuits such as those in Sec. V, one needs to use computer aided tools. We found it helpful to put several of the larger circuits through an initial round of automated reduction with PyZX, an open source PYTHON library designed to reduce, validate, and visualize $\mathrm{ZX}$-calculus diagrams [36]. PyZX applies a recursive, greedy algorithm [37]. Though the strategy of the PyzX library achieves significant reductions, it does not necessarily take into account the additional gate costs mentioned above (for instance, the reduced graphs of PyzX tend to have many Hadamard gates). Nonetheless, having reduced the overall graph size, it became feasible in isolation to tackle the $\pi / 4, \pi / 8$, and Hadamard gates by hand.

\section{A. Translating from the $\mathrm{ZX}$-calculus to the $3 \mathrm{D}$ topological representation}

Having obtained a reduced ZX-calculus graph, we are faced with the task of translating this result back into a 3D topological encoded quantum circuit. In contrast to planar lattice surgery [32], the problem of correspondence between elements presents a problem unique to defect qubits: the basis of a merge or split (present in red and green spiders) is determined by the type of defect (primal or dual), so that some conversion must take place between adjacent $\mathrm{ZX}$ nodes in distinct bases. To overcome this challenge we propose a new interpretation of the ZX-graph: for braided logic, we view the nodes of a ZX-graph as qubits (with red 


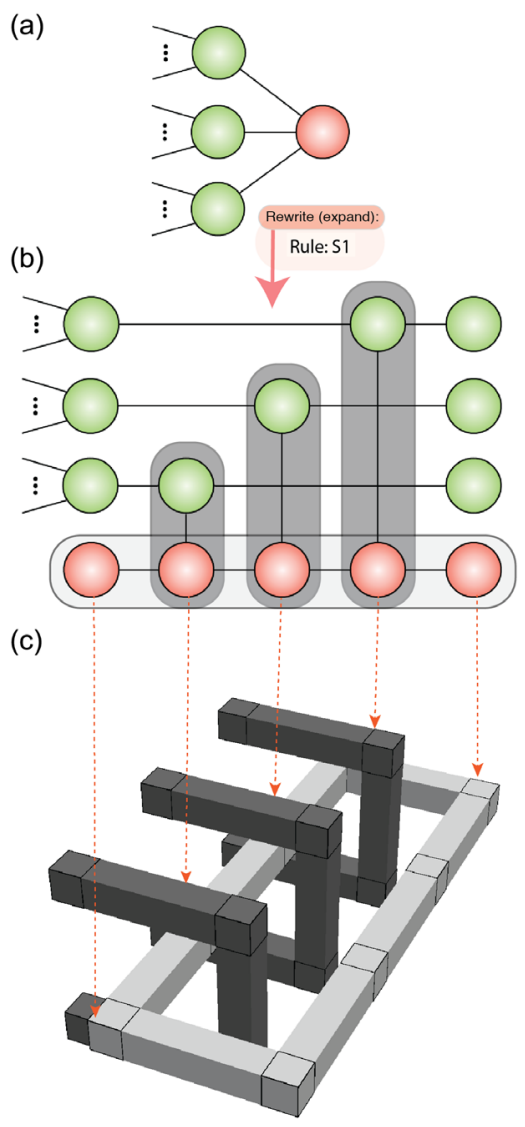

FIG. 5. Translation example. (a) Partial ZX-circuit illustrating a typical connected red spider node. (b) Expansion of the same circuit through the application of the $\mathrm{S} 1$ rule leading to a set of CNOT gates, initializations, and measurements. CNOT gate identification allows us to reduce the number of merge and split operations (and associated qubits) through braiding. (c) Associated 3D encoded circuit where the primal defect is identified with the red spider node. The identification of spider nodes with closed loops allows the nodes of the ZX-graph to be interpreted as qubits (in lieu of edges).

nodes as primal defect pairs and green nodes as dual defect pairs), edges between nodes of distinct colors as braided interactions between these qubits (see Fig. 5), and edges between nodes of the same color as junctions [38]. Our new interpretation is possible only because the ZX-calculus assumes positive-parity measurements at merge points (or applies corrections), such that parities propagate onto the output. Note that, due to constraints on interdefect distances, the number of edges still forms a lower bound on the surface area enclosed by defect loops. We do suspect, however, that the computational cost associated with problem of defect arrangement should be reduced along with the number of qubits.

Considering all nodes of degree 1 or 2 as local operations or state injections, several axioms (or rules) of the ZXcalculus (see Fig. 4) now take on familiar forms as transformations of topologically encoded circuits. For example, the $\pi$-copy rule describes the propagation of correlation surfaces [19], while the Hopf rule for edge pairs between adjacent nodes becomes the parity of the winding number in the braided circuit. The S2 rule describes the triviality of a pair of operations swapping back and forth between primal and dual qubits. The B2 rule is nontrivial, though we find the structure of this element in Fig. 12 of Ref. [38].

\section{EXEMPLAR CIRCUITS}

We are now equipped to apply the compression method described in Sec. III to some relevant exemplary circuits. For this we have chosen two important elements of many large quantum circuits: $Y$-state preparation for the application of the $\pi / 4$ gate and $A$-state distillation for the application of the $\pi / 8$ gate. Though the search for lowoverhead fractional phase gates is ongoing [39-45], we focus here on the simple cases where the 15-qubit ReedMuller code is used for A-state distillation and Steane's code for the $Y$ state [46]. We note that recent work has proposed a $\pi / 4$ gate implementation to circumvent the need for distillation [21]. These circuits have not only been chosen due to the critical importance of these states in surface-code quantum computation but also because they allow us to compare our method to the one presented in Ref. [5].

The surface code allows the $\sigma_{X}, \sigma_{Z}$, Hadamard, and CNOT gates transversally. To achieve a universal quantum gate set we may add the $\pi / 8$ gate. The current method of achieving this gate was proposed by Bravyi and Kitaev in 2005 [47] and makes use of gate teleportation [48-50]. The key is that the ancillary state containing information about the gate is a known state, the $A$ state. This means we can use an error detection protocol to improve its fidelity to levels consistent with other logical qubits before applying its effects via teleportation. Such ancillary states are referred to as magic states. Importantly, Bravyi and Kitaev showed that only Clifford gates $\{H, \pi / 4, \mathrm{CNOT}\}$ were required in the distillation protocol. Further, while $\pi / 4$ gates are required for $A$-state distillation, only $\sigma_{z}$ gates are required to play the equivalent role for $\pi / 4$ gates in $Y$-state distillation.

The teleported gates are achieved in 3D topological circuits via state injection. This procedure measures the two-qubit $\hat{Z}_{1} \hat{Z}_{2}$ operator through a merge operation. On a parity result of zero the phase is inherited by the target, while on a parity result of one a corrective phase rotation with double the target angle is required. To perform the projective $\hat{Z}_{1} \hat{Z}_{2}$ measurement in a $3 \mathrm{D}$ topological quantum circuit, the single qubit on which a faulty $\pi / 4$ or $\pi / 8$ gate is initially applied must be "grown" $[5,8,20,51]$ into a defect pair so that a merge operation with the target can be performed. This growth is represented diagrammatically as a pair of pyramidal structures emerging in opposite directions from a point. In this paper, we use the convention that such pyramidal structures are colored red for $\pi / 8$ gates 


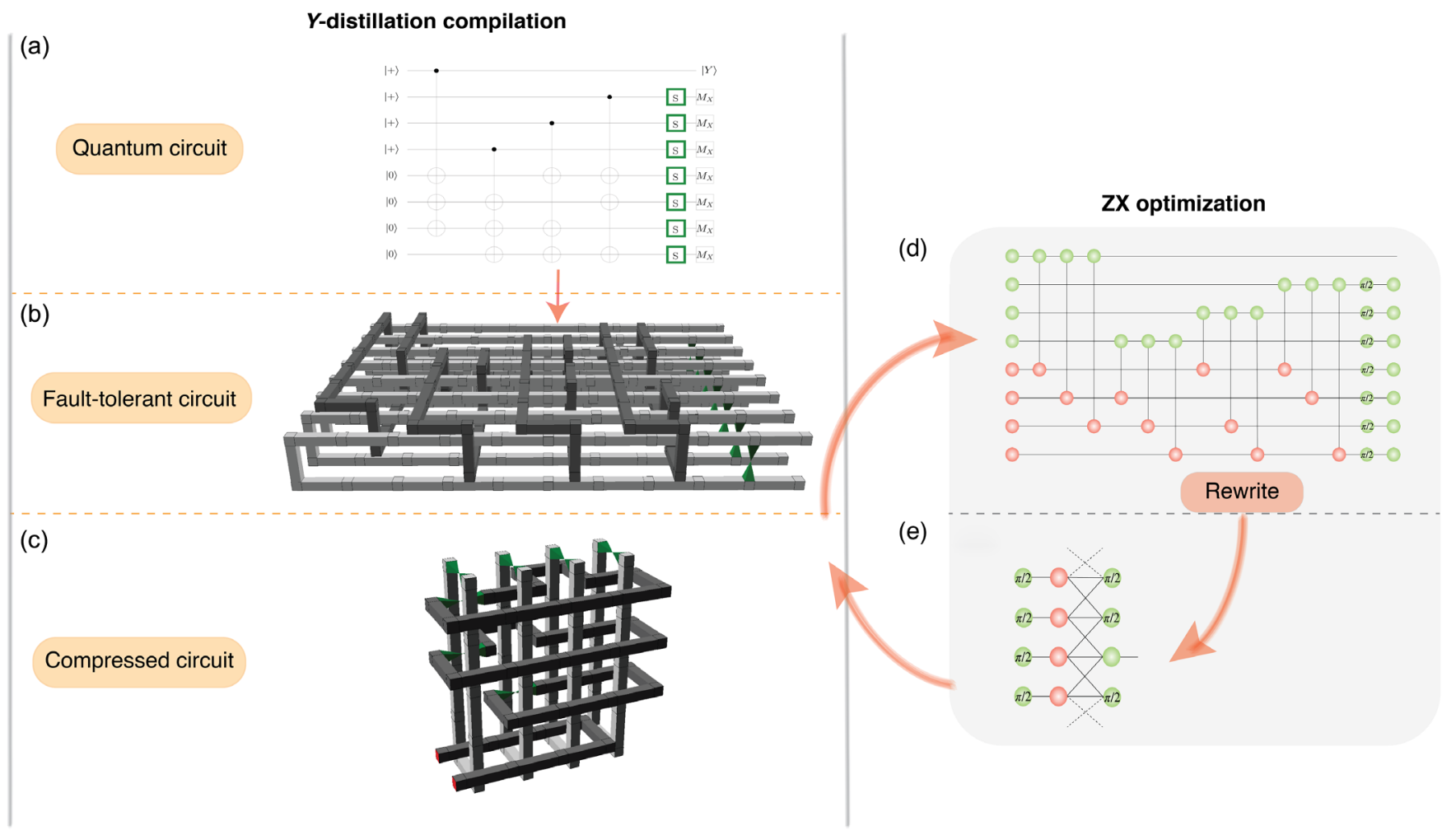

FIG. 6. Compilation and optimization of the $Y$-distillation circuit. (a) Initial circuit in the diagram form. (b) Initial circuit in the braided representation. (c) Reduced circuit in the braided representation after applying our compression method. (d) Initial circuit in the ZXcalculus representation. (e) Reduced circuit in the ZX-graph representation. Note that the dashed lines indicate periodic boundaries.

and green for $\pi / 4$ gates. The $\pi / 8$ gates may require trailing $\pi / 4$ corrections. Space has been allowed for these corrections in the circuits to follow, though we do not show them explicitly.

\section{A. $|Y\rangle$ distillation circuit}

In surface-code quantum computing, the $Y$ state, $\sqrt{2}|Y\rangle=|0\rangle+e^{i \pi / 2}|1\rangle$, is used to implement the phase $(\pi / 4)$ gate via gate teleportation. Even though this gate does not form part of the universal set of gates, a possible phase-gate correction is required for every application of the $\pi / 8$ gate. Some subcircuit for $Y$-state preparation is therefore expected to be ubiquitous in quantum computing, and its reduction is desirable. The preparation of the highfidelity $Y$ state may be achieved either directly [21] or via one of a series of distillation codes. For simplicity and in aid of our explanation, here we focus on the most straightforward distillation approach, the direct application of the Steane quantum error correction code in a new logical layer [see Fig. 6(a)].

Mapping the $Y$-state distillation circuit to the 3D representation is straightforward, as it only involves CNOT and phase gates [see Fig. 6(b)]. CNOT gates are represented as dual defects braiding the target primal qubits, while phase gates are performed through state injection, as described above. Similarly, we can represent the original circuit in the ZX-language [see Fig. 6(d)]. CNOT gates are then represented as a connected pair of green (control) and red (target) nodes between appropriate qubit wires, while phase gates are represented as green nodes with phase labels.

Through application of rules $\mathrm{S} 1$ and $\mathrm{S} 2$, we are able to reduce the $\mathrm{ZX}$-diagram for the $Y$-state distillation circuit to that of Fig. 6(e). It is important not to break the protection of the Steane code by overoptimizing the ZX-diagram. To ensure the code structure is maintained, we can validate the code through the application of the $\pi$-copy rule. This rule allows us to easily visualize how $\pi$ errors propagate forward through the diagram in time [13] to check whether the code still provides a unique syndrome for each correctable error. Once the ZX-diagram is reduced, and aided by the interpretation introduced in Sec. III A, we are ready to translate the reduced $|Y\rangle$ distillation circuit back to the braided representation.

The ZX-diagram does not provide any information about the time or space direction of the subdiagram that sits between the inputs and outputs. Therefore, nodes can be arranged anywhere in a $2 \mathrm{D}$ plane so long as the connectivity is preserved. When translating to the $3 \mathrm{D}$ representation, this means we must decide how to arrange the braided components in space and time, selecting a causal structure. In general, these decisions will depend on the relative costs associated with time and physical qubit number. 
Translating the reduced $Y$-state distillation circuit, we obtain the 3D topological circuit shown in Fig. 6(c). The dimensions of this circuit in units of the code distance $d$ are $2 \times 4 \times 4$, for a total volume of 32 . This compares with a direct translation from the circuit diagram [Fig. 6(a)], for which we obtain a circuit of dimensions $1.5 \times 8 \times 9$, for a total volume of 108 . We note that, though the circuit is too small to give a sense of the general trade-off between qubits and time, we are still free to reorient the circuit to emphasize one or the other resource. In this case, simple reorientation allows either a 4-time-step, 8-qubit circuit or a 2-time-step, 16-qubit circuit.

The $Y$-state distillation circuit is a natural initial test for any reduction procedure, and in 2012 Fowler and Devitt [9] addressed it to propose the 3D transformation rule "bridging." The size of the circuit thus obtained was $2 \times 1.5 \times 3$ for a volume of 9 . Clearly this is smaller than the 32-volume circuit we were able to obtain, and we now address the sources of this discrepancy.

The first of the differences between the two methods arises in the encoding of qubits in 3D space; braided circuits typically encode qubits in local defect pairs, forming a subsystem code and ignoring additional "gauge" degrees of freedom. This encoding decision is enforced for all transformations from a ZX-graph to a 3D circuit, just as it would be for any other intermediate representation. The 3D bridging transformation proposed by Fowler and Devitt, however, breaks this restriction, and is therefore able to encode a greater amount of information in the same space. It remains an interesting open question what restrictions apply to the use of these additional degrees of freedom in translation from the ZX-language, and we intend to investigate this point further in a forthcoming paper.

The second difference between the result of Ref. [9] and our own is that we have placed limits on the circuit reduction that were not present in their work. These limits maintain the independence of error syndromes for the Steane code, and preserve the basis of the output state. Following a set of single-qubit operators across the result of Ref. [9], we draw two important conclusions: firstly, errors from injected gates no longer result in independent syndromes, so that the order of detectable operations and the logical gate fidelity of the Steane code are reduced; secondly, the final state is in a basis orthogonal to the $|Y\rangle$ state - a consequence of swapping the basis of the codeso that a trailing Hadamard gate and additional volume will be necessary. This difference highlights the importance of the procedure provided by the ZX-calculus for verifying error independence, and we expect this to become more relevant as circuit sizes increase.

\section{B. $|A\rangle$ distillation circuit}

Having established that the ZX-calculus can be used to reduce and verify the $Y$-state distillation subcircuit, let us now turn to the more expensive problem of $A$-state distillation for the $\pi / 8$ gate. The $A$ state, $\sqrt{2}|A\rangle=$ $|0\rangle+e^{i \pi / 4}|1\rangle$, is used to implement the $\pi / 8$ gate, the most common final element of a universal quantum gate set incorporating also the Clifford gates (CNOT, $X, H$, and $S$ ). Once again, there are a number of distillation codes that could be chosen, and for simplicity we focus on the most conceptually simple: the direct application of the 15-qubit quantum Reed-Muller code, depicted in Fig. 7(a).

The ZX-calculus axioms shown in Fig. 4 are complete only for the Clifford gates. To achieve a complete set of axioms for the Clifford $+T$ gate set, several additions must be made $[30,31]$. While it would be possible to identify structural identities corresponding to these additional axioms, their complexity makes their manual discovery and application difficult. Fortunately for our example, we are forearmed with knowledge of the quantum Reed-Muller code structure and we know that it will not be possible to reduce the number of $T$ gates without breaking the structure of the code, and so in this case we need not attempt to apply the remaining axioms. Once again, through application of $\mathrm{S} 1$ and $\mathrm{S} 2$, we have been able to considerably reduce the complexity of the ZX-diagram for the $|A\rangle$ distillation circuit, as shown in Fig. 7(e). After translating the ZXreduced $A$-state distillation circuit back into the $3 \mathrm{D}$ representation, we obtain a reduced circuit with a total volume of 125. From an initial volume of 360 , the compression of this circuit yields an outstanding compression rate of $65 \%$.

As for the $Y$ state in Sec. IVA, a reduced 3D structure for the $A$-state distillation circuit was given in Ref. [9]. For this circuit, however, the reduction that was achieved with the topology-preserving transformations in 3D space was more limited, achieving a reduced volume of $15.5 \times 5.5 \times 2.5=213.125$, or a $32 \%$ reduction. In stark contrast to the previous example of $Y$-state distillation, the 125 -volume circuit we obtain for $A$-state distillation is a further $41 \%$ smaller than the previously reduced circuit. We note that the striking difference between these two compression rates highlights the capabilities of the ZX-calculus as an intermediate language for braided circuit optimization. The limited reduction attained when the optimization had been performed in the 3D representation was a result of the presence of $\pi / 8$ gates, which served to partition the circuit into isolated regions, limiting applicable structural transformations.

\section{BENCHMARKING WITH GENERAL CIRCUITS}

So far we have seen the application of the ZX-calculus to circuit reduction and verification in two examples: the $Y$-state and $A$-state distillation circuits. To show the generality of our approach, we have selected a small set of arbitrary Clifford $+T$ circuits as benchmarks to assess the performance of our circuit optimization method. In Table I, in addition to the distillation circuits, we outline four circuits with their respective original volumes in the $3 \mathrm{D}$ representation $\left(\mathrm{Vol}_{\text {init }}\right)$ and the reduced volume after 


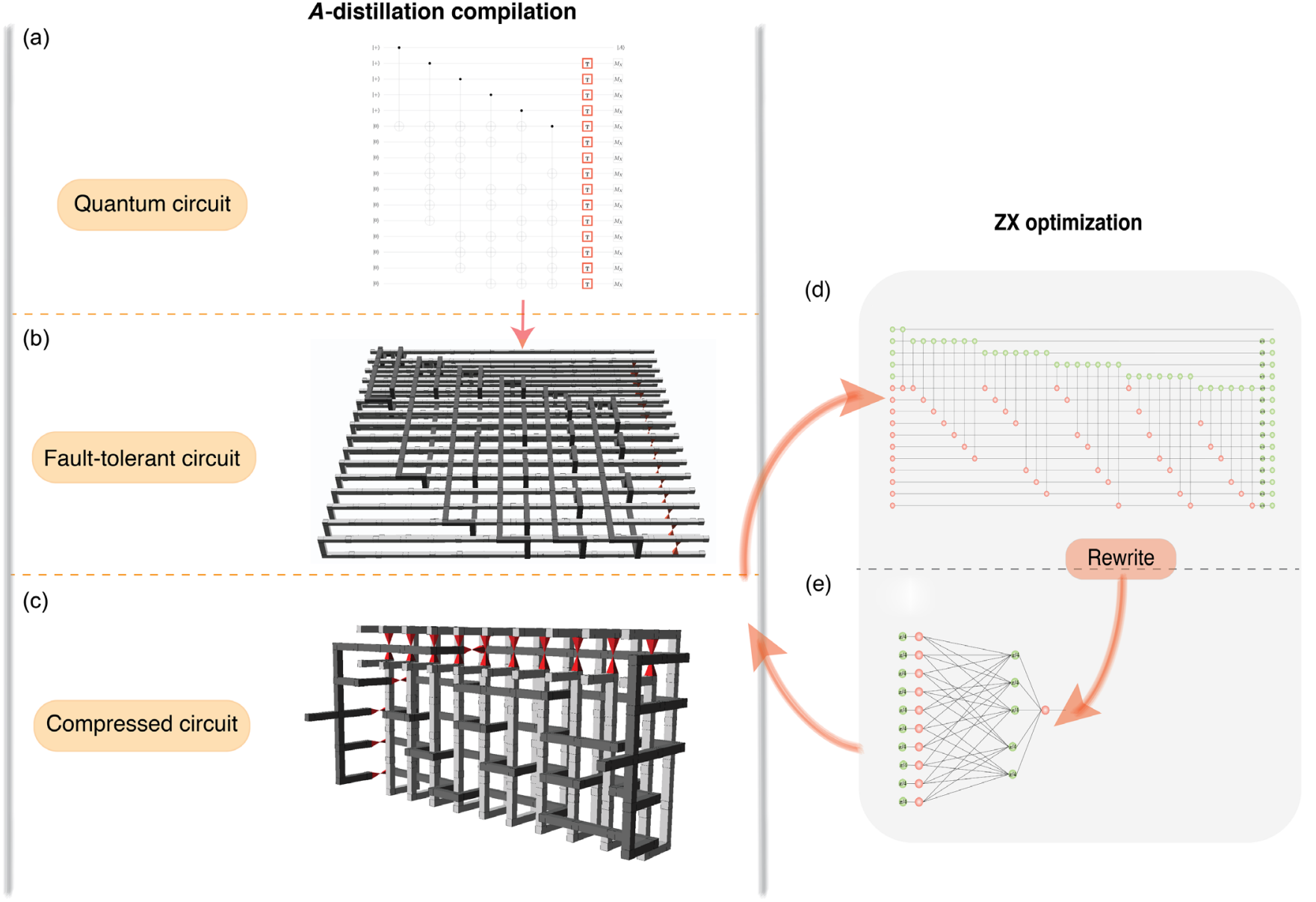

FIG. 7. Compilation and optimization of the $A$-distillation circuit. (a) Initial circuit in the diagram form. (b) Initial circuit in the braided representation. (c) Reduced circuit in the braided representation after applying our compression method. (d) Initial circuit in the ZXcalculus representation. (e) Reduced circuit in the ZX-graph representation. Note that the dashed lines indicate periodic boundaries.

application of ZX-calculus $\left(\mathrm{Vol}_{\mathrm{opt}}\right)$. Our results show compression percentages of up to $\sim 77 \%$, giving a very promising indication of the viability of our method for braided circuit compression against other approaches. The corresponding 3D structures of such circuits before and after optimization are shown in Fig. 8. To account for fault tolerance, the volume costs of magic state distillation for $T$ gates should be added to the total volume of these additional circuits. In such cases, that sum would represent an

TABLE I. Volumes before and after optimization for a set of circuits from the Pyzx circuit database [36].

\begin{tabular}{lcc}
\hline \hline \multicolumn{3}{c}{ Benchmarks } \\
\hline Circuit & Vol $_{\text {init }}$ & $\mathrm{Vol}_{\text {opt }}$ \\
\hline$Y$-distillation & 108 & $32(-70.3 \%)$ \\
$A$-distillation & 360 & $125(-65 \%)$ \\
barenco-tof-3-after-light & 510 & $262.125(-48.6 \%)$ \\
mod-5-4-before & 555 & $119.625(-77.4 \%)$ \\
tof-4-before & 882 & $420(-52.4 \%)$ \\
vbe-adder-3-before & 1995 & $563.5(-72 \%)$ \\
\hline \hline
\end{tabular}

upper bound, as the reduction of a larger ZX-graph including the distillation circuits for the $S$ and $T$ gates could potentially yield a smaller structure than the simple sum of the separate volumes.

\section{FURTHER PACKING AND LATTICE SURGERY}

While most of our compression is attained at the level of reduction of the ZX-diagram, when translating back to the $3 \mathrm{D}$ representation we have some freedom in the way we arrange the braids in space. This arrangement depends on their connectivity, so that an optimal packing of the structure allows us to further reduce the volume. In addition, we can reinterpret some parts of our reduced ZX-graph as merge and split operations between defects analogous to lattice surgery. Whether it is advantageous for volume reduction to interpret a node as a braid loop or as such a defect-surgery operation depends on the arrangement of the surrounding geometric structure. Note that the ZX spider rule (S1) allows us to divide a single node into "braid" and "surgery" regions. The surgery approach was 

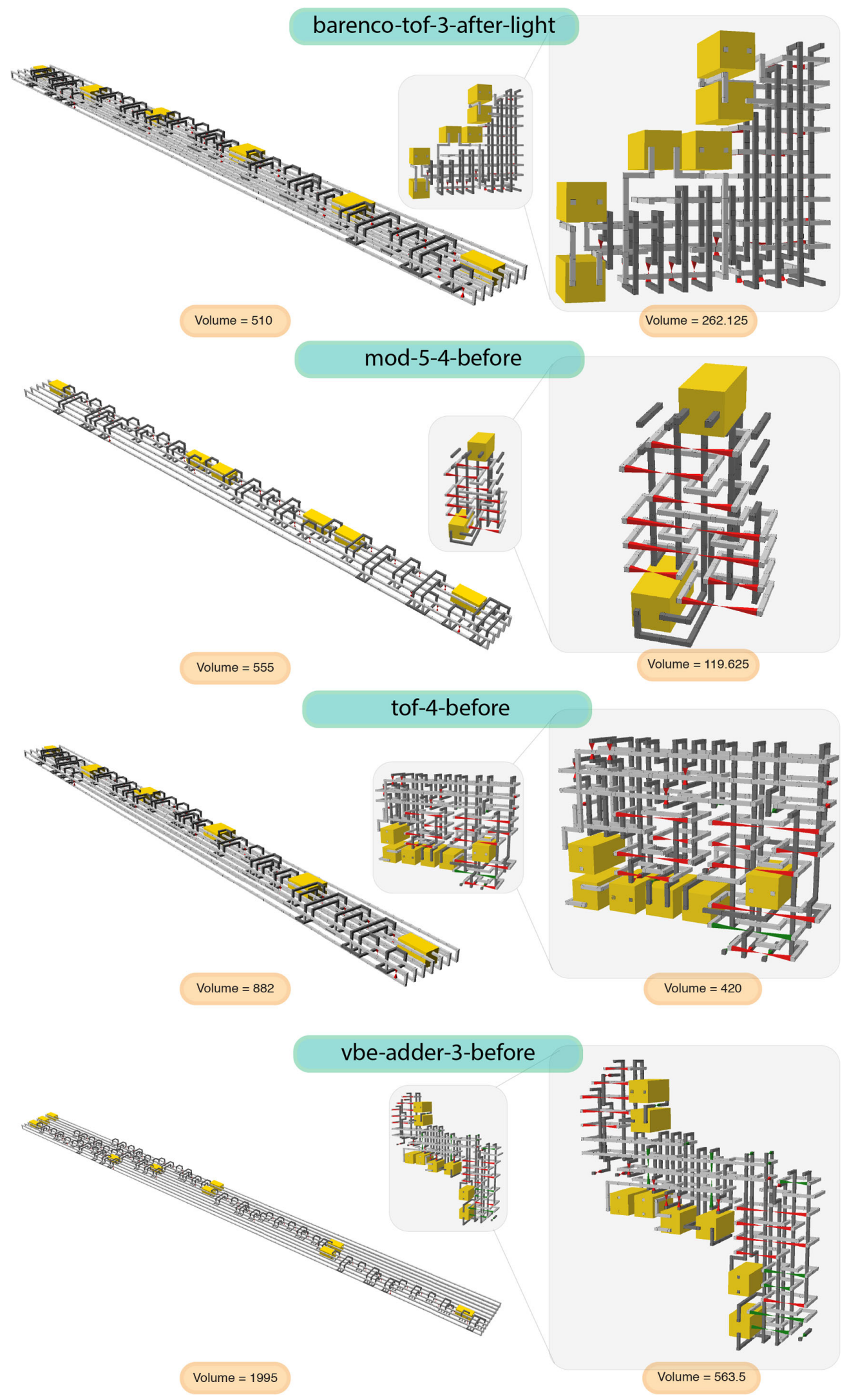

FIG. 8. 3D structures of the benchmarked circuits before and after compression and their respective volumes. We show the scaled structure to be compared with the initial nonreduced circuit and then an enlargement for detail. 
(a)

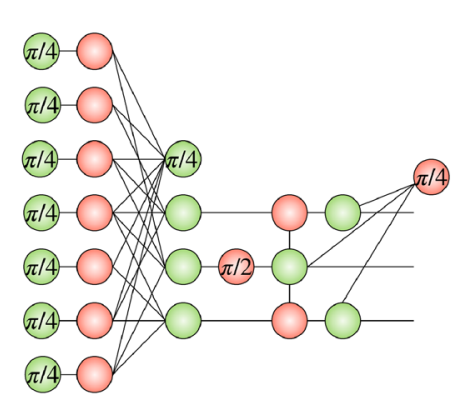

(b)

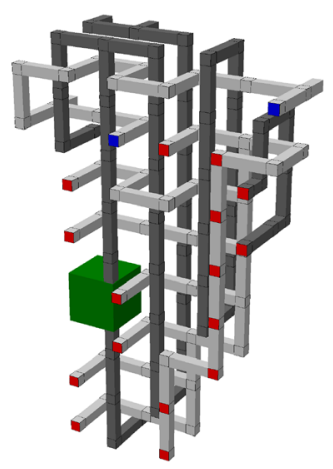

FIG. 9. (a) Reduced ZX-diagram for the $|C C Z\rangle$-catalyzed $2|T\rangle$ factory from Ref. [52]. (b) Corresponding 3D structure after packing and applying the hybridized approach for braiding and defect-surgery discussed in Sec. VI. Here we follow Fig. 13 of Ref. [52] in substituting a green box for a single-qubit Clifford gate. We have marked the inputs of $T$ states in red and the recycled $T$ state for catalysis in blue.

in fact applied in the compression of the $|A\rangle$ distillation circuit from Fig. 7, and is implicit in our decision to treat degree-one and degree-two nodes as local operations or state injections.

To date, the most efficient $T$-gate distillation circuit is the $|C C Z\rangle$-catalyzed $2|T\rangle$ lattice-surgery-based circuit depicted in Fig. 18 of Ref. [52], with a volume of 120 [53]. After we apply our compression method to the same initial circuit and pack the resultant structure, we get a volume of 58 (see Fig. 9). For a fair comparison between the distance measures of these two approaches, we should rescale our volume by a factor of $25 / 16$ to account for the defect diameter, so that we obtain 91 . Such numbers tell us that braid-based surface-code computation should not be lightly dismissed in favor of lattice surgery, despite previous suggestions of increased storage costs [9]. Instead, a hybrid approach to the compilation of fault-tolerant quantum circuits seems promising.

\section{CONCLUSIONS}

Although quantum computers are still far from maturity, early proof-of-principle experiments demonstrating quantum advantage have already been achieved [4]. This sets the focus now on the pursuit of quantum practicality for largescale applications. To achieve this, quantum error correction must be incorporated, adding large overheads in terms of qubit number and time relative to preceding near-term devices. In this context it becomes critical to reduce the size of fault-tolerant quantum circuits during compilation.

We have presented a new approach to the problem of compressing braided quantum circuits using the ZXcalculus as an intermediate language. The standard interpretation of the ZX-graph identifies edges as qubits and nodes as operation tensors. To date, considerations of cluster-state quantum computing, even involving defects,

have followed this approach [33]. In this work, we identify that in inverting the standard interpretation we still describe a valid quantum circuit. But in this case, the ZX-calculus describes braided, rather than measurement-based logic.

The use of this representation not only allows easy manipulation of the circuit but also makes straightforward the verification of the structure of higher-level errorcorrection codes as well as the correctness of the computation for Clifford subcircuits. We have applied our compression method to arbitrary Clifford $+T$ circuits, including ubiquitous magic state distilleries, reaching reduction percentages of up to $\sim 77 \%$. Further, in addition to our observed volume reductions in units of distance, the smaller size of the circuits should reduce the required logical error rate and the associated code distance itself. Quantities for the code distance cannot be estimated without a knowledge of the specific error channels and the structure of the larger quantum computation in which the circuit is embedded. However, we expect significant resource reductions at the physical qubit level [54], especially for those subcircuits such as magic state distilleries for which errors are heralded and the circuits can be rapidly repeated.

It is one thing that logical information can be encoded and manipulated in the surface code in two different ways. It is quite another to find that the ZX-calculus is a neutral representation of the correspondence between them at the level of the individual logical operations. In the topological quantum computation literature, the question of encoding has appeared to be settled since about 2018 [55]. Subsequent work designing efficient fault-tolerant circuits has assumed the lattice-surgery approach [14]. Crucially, neither of our interpretations of the ZX-calculus for surface-based topological models excludes the other, and thus the ZX-calculus is a language capable of unifying both expressions of computation in the same diagram. With compression rates as reported in this paper, overheads of braided, defect-based circuits are comparable, and even lower, to those obtained for their lattice-surgery counterparts. With the ability to translate between these models of computation, our work not only has the potential to resurrect braiding as a viable prospect, but to propose a new approach for computation based on hybrid circuits, an option not previously considered outside of single-qubit gate implementations. Indeed, in our own results we have found that the best route has consisted of a hybridization of these approaches.

Finally, prior work has focused heavily on the reduction of a small number of common subcircuits, for which efforts are expected to show progressively diminishing returns. By contrast, our approach considers larger circuits in their entirety, making reduction of the remaining $\sim 50 \%$ of these circuits accessible. This work therefore opens a new direction for the compilation of surface-code-based quantum computation. To generalize the applicability and 
scalability of our approach, in future work we plan to explore strategies and tools to automate the entire set of compilations and reductions.

\section{ACKNOWLEDGMENTS}

We thank Koki Suetsugu, Kunihiro Wasa, and Yu Yokoi for interesting discussions, as well as Austin Fowler and Craig Gidney for helpful comments on an early manuscript. This work was supported in part by the Japanese MEXT Quantum Leap Flagship Program (MEXT Q-LEAP), Grant No. JPMXS0118069605.

Note added.-Recently, we became aware of similar work being pursued by Alexandru Paler [56].

[1] D. Deutsch, Quantum Theory, the Church-Turing Principle and the Universal Quantum Computer, Proc. R. Soc. A 400, 97 (1985).

[2] P. W. Shor, Polynomial-Time Algorithms for Prime Factorization and Discrete Logarithms on a Quantum Computer, SIAM J. Comput. 26, 1484 (1997).

[3] L. K. Grover, A Fast Quantum Mechanical Algorithm for Database Search, in Proceedings of the Twenty-Eighth Annual ACM Symposium on Theory of Computing (STOC '96) (Association for Computing Machinery Press, New York, 1996)

[4] F. Arute et al., Quantum Supremacy Using a Programmable Superconducting Processor, Nature (London) 574, 505 (2019).

[5] A. G. Fowler, M. Mariantoni, J. M. Martinis, and A. N. Cleland, Surface Codes: Towards Practical Large-Scale Quantum Computation, Phys. Rev. A 86, 032324 (2012).

[6] S. B. Bravyi and A. Yu. Kitaev, Quantum Codes on a Lattice with Boundary, arXiv:quant-ph/9811052.

[7] M. H. Freedman and D. A. Meyer, Projective Plane and Planar Quantum Codes, Found. Comput. Math. 1, 325 (2001).

[8] E. Dennis, A. Kitaev, A. Landahl, and J. Preskill, Topological Quantum Memory, J. Math. Phys. (N.Y.) 43, 4452 (2002).

[9] A. G. Fowler and S. J. Devitt, A Bridge to Lower Overhead Quantum Computation, arXiv:1209.0510.

[10] C. Horsman, A. G. Fowler, S. Devitt, and R. Van Meter, Surface Code Quantum Computing by Lattice Surgery, New J. Phys. 14, 123011 (2012).

[11] A. Paetznick and A. G. Fowler, Quantum Circuit Optimization by Topological Compaction in the Surface Code, arXiv:1304.2807.

[12] B. Coecke, Quantum Picturalism, Contemp. Phys. 51, 59 (2010).

[13] N. Chancellor, A. Kissinger, J. Roffe, S. Zohren, and D. Horsman, Graphical Structures for Design and Verification of Quantum Error Correction, arXiv:1611.08012.

[14] D. Litinski, A Game of Surface Codes: Large-Scale Quantum Computing with Lattice Surgery, Quantum 3, 128 (2019).
[15] T. M. Stace, S. D. Barrett, and A.C. Doherty, Thresholds for Topological Codes in the Presence of Loss, Phys. Rev. Lett. 102, 200501 (2009).

[16] T. M. Stace and S. D. Barrett, Error Correction and Degeneracy in Surface Codes Suffering Loss, Phys. Rev. A 81, 022317 (2010).

[17] S. D. Barrett and T. M. Stace, Fault Tolerant Quantum Computation with Very High Threshold for Loss Errors, Phys. Rev. Lett. 105, 200502 (2010).

[18] H. J. Briegel, D. E. Browne, W. Dür, R. Raussendorf, and M. Van den Nest, Measurement-Based Quantum Computation, Nat. Phys. 5, 19 (2009).

[19] A. Paler, Design Methods for Reliable Quantum Circuits, Ph.D. thesis, Universität Passau, 2015.

[20] A. G. Fowler, A. M. Stephens, and P. Groszkowski, HighThreshold Universal Quantum Computation on the Surface Code, Phys. Rev. A 80, 052312 (2009).

[21] B. J. Brown, K. Laubscher, M. S. Kesselring, and J. R. Wootton, Poking Holes and Cutting Corners to Achieve Clifford Gates with the Surface Code, Phys. Rev. X 7, 021029 (2017).

[22] A. Paler, S. Devitt, K. Nemoto, and I. Polian, Synthesis of Topological Quantum Circuits, in Proceedings of the 2012 IEEE/ACM International Symposium on Nanoscale Architectures (NANOARCH '12) (Association for Computing Machinery Press, New York, 2012).

[23] Y. Ding, A. Holmes, A. Javadi-Abhari, D. Franklin, M. Martonosi, and F. Chong, Magic-State Functional Units: Mapping and Scheduling Multi-Level Distillation Circuits for Fault-Tolerant Quantum Architectures, in Proceedings of the 51st Annual IEEE/ACM International Symposium on Microarchitecture, 2018 (MICRO51) (IEEE, New York, 2018).

[24] A. Paler, A. G. Fowler, and R. Wille, Online Scheduled Execution of Quantum Circuits Protected by Surface Codes, Quantum Inf. Comput. 17, 1335 (2017).

[25] A. G. Fowler, Time-Optimal Quantum Computation, arXiv: 1210.4626.

[26] Reversible Computation, edited by J. Krivine and J.-B. Stefani (Springer International Publishing, Switzerland, 2015).

[27] A. Paler and S. J. Devitt, Specification Format and a Verification Method of Fault-Tolerant Quantum Circuits, Phys. Rev. A 98, 022302 (2018).

[28] B. Coecke and R. Duncan, Interacting Quantum Observables, Automata, Languages and Programming (Springer, Berlin, 2008), pp. 298-310.

[29] B. Coecke and R. Dunca, Interacting Quantum Observables: Categorical Algebra and Diagrammatics, New J. Phys. 13, 043016 (2011).

[30] M. Backens, The ZX-Calculus is Complete for the SingleQubit Clifford $+T$ Group, in Proceedings of the 11th workshop on Quantum Physics and Logic (QPL 2014), EPTCS 172, pp. 293-303.

[31] B. Coecke and Q. Wang, ZX-Rules for 2-Qubit Clifford $+T$ Quantum Circuits, arXiv:1804.05356.

[32] N. de Beaudrap and D. Horsman, The ZX Calculus Is a Language for Surface Code Lattice Surgery, Quantum 4, 218 (2020). 
[33] C. Horsman, Quantum Picturalism for Topological ClusterState Computing, New J. Phys. 13, 095011 (2011).

[34] A. Kissinger and J. van de Wetering, Reducing the Number of Non-Clifford Gates in Quantum Circuits, Phys. Rev. A 102, 022406 (2020).

[35] M. Backens, Making the Stabilizer ZX-Calculus Complete for Scalars, in Proceedings of the 12th International Workshop on Quantum Physics and Logic (QPL 2015), Electronic Proceedings in Theoretical Computer Science, edited by C. Heunen, P. Selinger, and J. Vicary (Open Publishing Association, 2015), Vol. 195, pp. 17-32, https:// doi.org/10.4204/EPTCS.195.2.

[36] Quantomatic/pyzx, https:/github.com/Quantomatic/pyzx/ tree/master/circuits.

[37] A. Kissinger and J. van de Wetering, Pyzx: Large Scale Automated Diagrammatic Reasoning, in Electronic Proceedings in Theoretical Computer Science 318, (2020), pp. 229-241.

[38] R. Raussendorf, J. Harrington, and K. Goyal, Topological Fault-Tolerance in Cluster State Quantum Computation, New J. Phys. 9, 199 (2007).

[39] A. M. Meier, B. Eastin, and E. Knill, Magic-State Distillation with the Four-Qubit Code, arXiv:1204.4221.

[40] S. Bravyi and J. Haah, Magic-State Distillation with Low Overhead, Phys. Rev. A 86, 052329 (2012).

[41] C. Jones, Multilevel Distillation of Magic States for Quantum Computing, Phys. Rev. A 87, 042305 (2013).

[42] C. Jones, Low-Overhead Constructions for the FaultTolerant Toffoli Gate, Phys. Rev. A 87, 022328 (2013).

[43] C. Jones, Distillation Protocols for Fourier States in Quantum Computing, arXiv:1303.3066.

[44] B. Eastin, Distilling One-Qubit Magic States into Toffoli States, Phys. Rev. A 87, 032321 (2013).

[45] A. Paetznick and B. W. Reichardt, Universal Fault-Tolerant Quantum Computation with Only Transversal Gates and Error Correction, Phys. Rev. Lett. 111, 090505 (2013).
[46] It is worth emphasizing that these circuits do not represent the state of the art in terms of overall cost; we select them here primarily for the purpose of comparative reduction.

[47] S. Bravyi and A. Kitaev, Universal Quantum Computation with Ideal Clifford Gates and Noisy Ancillas, Phys. Rev. A 71, 022316 (2005).

[48] C. H. Bennett, G. Brassard, C. Crépeau, R. Jozsa, A. Peres, and W. K. Wootters, Teleporting an Unknown Quantum State via Dual Classical and Einstein-Podolsky-Rosen Channels, Phys. Rev. Lett. 70, 1895 (1993).

[49] D. Gottesman and I. L. Chuang, Quantum Teleportation Is a Universal Computational Primitive, Nature (London) 402, 390 (1999).

[50] D. Gottesman and I. L. Chuang, Demonstrating the Viability of Universal Quantum Computation Using Teleportation and Single-Qubit Operations, Nature (London) 402, 390 (1999).

[51] A. G. Fowler and K. Goyal, Topological Cluster State Quantum Computing, Quantum Inf. Comput. 9, 721 (2009).

[52] C. Gidney and A. G. Fowler, Efficient Magic State Factories with a Catalyzed $|C C Z\rangle$ to $2|T\rangle$ Transformation, Quantum 3, 135 (2019).

[53] In Fig. 1 of Ref. [52], the authors suggest outer bounding boxes for the $C C Z$ factory and catalyzed 2-T circuit of $3 \times 6 \times 5.5=99$ and $4 \times 4 \times 6.5=104$, respectively, for a combined volume of 203. However, it is possible that some space may be saved when these components are packed or tiled within a larger circuit. To account for this, we use a highly conservative estimate for the volume, derived from Fig. 18 of Ref. [52]: we assign the $C C Z$ factory a volume of $2 \times 5 \times 6=60$ and the catalyzed $2-T$ circuit a volume of $3 \times 4 \times 5=60$, for a combined total of 120 .

[54] D. Litinski, Magic State Distillation: Not as Costly as You Think, Quantum 3, 205 (2019).

[55] A. G. Fowler and C. Gidney, Low Overhead Quantum Computation Using Lattice Surgery, arXiv:1808.06709.

[56] A. Paler (private communication). 